\title{
LA PRUEBA ES LIBERTAD, PERO NO TANTO: UNA TEORÍA DE LA PRUEBA CUASI-BENTHAMIANA ${ }^{1}$ \\ Proof in law is freedom but not so much: a quasi-Benthamian theory of the proof
}

Jordi Ferrer Beltrán

Fecha de recepción: 19 de abril de 2017

Fecha de aceptación: 04 de mayo de 2017

Sumario: 1. Introducción; 2. Los momentos de la actividad probatoria en el proceso judicial; 2.1. La conformación del conjunto de elementos de juicio o pruebas; 2.2. La valoración de los elementos de juicio o pruebas; 2.3. La adopción de la decisión sobre los hechos probados; 3. La crítica de Stein a la tradición racionalista 4. Reconsiderando las tesis de partida; 5. Referencias Bibliográficas.

${ }^{1}$ Agradezco los muy útiles comentarios de Susan Haack, Larry Laudan, Giovanni B. Ratti y Carmen Vázquez. 


\section{CÓMO SE CITA ESTE ARTíCULO (Normas APA-6)}

Ferrer Beltrán, Jordi (2017). La prueba es libertad, pero no tanto: Una teoría de la prueba Cuasi-Benthamiana. Revista Jurídica Mario Alario D'Filippo, IX (18), pág 150-169.

\section{RESUMEN}

La modesta pretensión de este trabajo es reevaluar un aspecto muy específico de la teoría de la prueba propia de la tradición racionalista para el que Bentham puede ser llamado en causa como precursor: la idea de que la prueba debe ser fundamentalmente libre y que cualquier interferencia del derecho procesal en ella debería ser limitada o eliminada. 


\section{INTRODUCCIÓN}

En el ámbito de estudio de la prueba, Jeremy Bentham es considerado uno de los principales precursores de la denominada tradición racionalista (especialmente en el mundo anglosajón, claro). Es más, se ha considerado, con razón, que la teoría benthamiana "constituye la más ambiciosa y completa teoría de la prueba en la historia del pensamiento jurídico" (Twining, 1994, p.39). Por ello, resulta de gran importancia hacer las cuentas con el pensamiento de Bentham si se quiere abordar el estudio de los problemas probatorios en el derecho.

No es mi intención, sin embargo, entrar en disquisiciones acerca de la mejor reconstrucción que pueda darse de esta o aquella tesis del autor; tampoco iniciar un debate con intérpretes más autorizados que yo de su pensamiento. La modesta pretensión de este trabajo es reevaluar un aspecto muy específico de la teoría de la prueba propia de la tradición racionalista para el que Bentham puede ser llamado en causa como precursor: la idea de que la prueba debe ser fundamentalmente libre y que cualquier interferencia del derecho procesal en ella debería ser limitada o eliminada. Bentham elabora una teoría prescriptiva de la prueba o, aún mejor para lo que nos importa, una teoría prescriptiva del derecho de la prueba, que es una parte más de su teoría general del derecho (Keeton y Marshall, 1948; Twining, 1985; Moreso, 1992)

La premisa básica de la que parte es simple y clara: la finalidad de la prueba en el proceso judicial es la averiguación de la verdad sobre lo ocurrido (Bentham, 1827; (Moreso, 1992; Twinnig, 1985-1994). Aunque, por supuesto, esta asunción de partida puede ser y ha sido muy discutida, creo que debe ser admitida salvo que se esté dispuesto a pagar altos costes en la reconstrucción del derecho (Ferrer, 2005). El objetivo de la averiguación de la verdad deriva, por otro lado, de la asunción por parte de Bentham de que la finalidad fundamental del derecho procesal es la de ser garantía de la correcta aplicación del derecho sustantivo (Betham, 1827, p.17-19). Finalidad que, a su vez, se divide en la necesidad de una doble garantía: asegurar que todos los infractores del derecho sean sancionados y que sólo ellos lo sean ${ }^{2}$. Y esto supone, evidentemente, la necesidad de que lo que se declare probado en el proceso coincida con la verdad de lo ocurrido: esto es, que los enunciados declarados probados sean verdaderos y los enunciados falsos no se declaren probados.

Siendo estos los objetivos, queda por determinar la metodología adecuada para alcanzarlos. Y en este aspecto, Bentham es extraordinariamente crítico con el derecho probatorio de su época (críticas en buena medida aplicables a nuestros sistemas procesales actuales). Para Bentham, la mejor forma de alcanzar ese objetivo es mediante una metodología o sistema "natural" de conocimiento (Twining, 1985; Hart, 1982; Postema, 1986), propia del sentido común y de la epistemología general (Bentham, 1827),

${ }^{2}$ Postema, 1986: 343, quien cita, a su vez, los Bentham Manuscripts in the University College, London Library. 
y no a través del denominado derecho probatorio que, en la Inglaterra de su época, estaba formado básicamente por un conjunto de reglas de exclusión que limitaban las posibilidades de conocimiento. Es más, Bentham (1838-43) considera que la justicia es algo bastante simple, "es lo mismo para un hombre que para otro: sólo los legisladores, aconsejados por los abogados, la han complicado y hecho trizas" (p.334). Resulta tan simple que el mejor método para administrar justicia es el que él denomina modelo doméstico, propio del "paterfamilias" en el trato de los hijos. Por ello, lo mejor que podría hacer el derecho en materia de prueba es callar, dejando reinar al principio general de la libertad probatoria (free proof) ${ }^{3}$.

Esta es una característica propia de la denominada tradición racionalista clásica en materia de prueba. Como afirma el propio Bentham (1823) en el Treatise on Judicial Evidence "en toda la trama de esta obra reaparece constantemente esta conclusión práctica: No hay que excluir ninguna prueba, ningún testimonio por el sólo temor de ser engañado" (p. 441). La cursiva es del autor). Por ello, es necesario "sustituir el principio de exclusión por el de suspicacia" ante las pruebas disponibles ${ }^{4}$. El "paterfamilias" que juzga la conducta de sus hijos tiende a aceptar todos los elementos de juicio puesto que la racionalidad indica que cuánto más abundante sea el conjunto de elementos de juicio disponible mayor será la probabilidad de determinar la verdad de lo ocurrido. Es este un corolario del reino de la epistemología en el proceso, buscado por Bentham, del método 'natural' de la prueba (Twinnig, 1994).

Y tampoco el derecho debe regular la valoración de la prueba (weight of evidence), puesto que ésta debe llevarla a cabo el juez, atendiendo a las circunstancias propias del caso y en aplicación del método de razonamiento 'natural'. Por ello, en conclusión, Bentham es absolutamente contrario a la existencia de reglas jurídicas vinculantes sobre la prueba: esta es la denominada "anti-nomian thesis" de Bentham, que ofrece una imagen clásica de la tradición racionalista de la prueba y que ha sido resumida por Twining (1994) en los siguientes términos:

El sistema jurídico no debe contener "ninguna norma que excluya testigos o pruebas; ninguna norma sobre el peso o el quantum de la prueba; ninguna norma vinculante sobre la forma de presentación de la prueba; ninguna restricción artificial sobre los interrogatorios o el razonamiento probatorio; ningún derecho de silencio ni privilegios de los testigos; ninguna restricción al razonamiento que no sean las propias del razonamiento práctico; ninguna exclusión de pruebas excepto si son irrelevantes o superfluas o si su presentación supone perjuicios, gastos o retrasos excesivos en las circunstancias del caso específico" $(\mathrm{p} 195)^{5}$.

\footnotetext{
${ }^{3}$ En este sentido, sostiene Twining que el derecho probatorio anglo-americano de los dos últimos siglos puede ser entendido como un serie de excepciones al principio general de free proof, de inspiración thayeriana. Vid. TWINING, 1994: 196. Vid. también DAMAŠKA, 1997: 149.

${ }^{4}$ Bentham, 1823 : 442. En la misma línea puede verse de forma reciente, por todos, Laudan, 2006: 122, 124.

${ }^{5}$ La tarea del legislador, pues, no es la de dictar reglas imperativas sino simplemente instrucciones orientativas acerca de la valoración
} 
La recepción de estas tesis de Bentham, tanto en el área de common law como de civil law, ha conllevado interpretaciones en términos claramente abolicionistas del derecho probatorio. Un caso muy claro es, en la cultura jurídica de lengua castellana, el de Sentís Melendo (1979) quien afirma que:

Derecho y probatorio, ese sustantivo y ese adjetivo, son términos antagónicos. La prueba es libertad; $y$ en el momento en que el derecho intenta someterla a normas rígidas, deja de ser prueba, para convertirse, si no en una caricatura, en algo que ya no es prueba" (p.336).

Yen términos parecidos en cuanto al análisis, que no en cuanto a la propuesta abolicionista, puede entenderse el muy conocido pasaje de Carnelutti (1947) "basta un límite mínimo a la libertad de búsqueda del juez para que el proceso de búsqueda de la verdad degenere en un proceso formal de fijación; en otros términos, la medida del límite es indiferente para la naturaleza del proceso" (p.33-4). A Sentís Melendo y Carnelutti les separa muy claramente la apuesta posterior al análisis: el primero propugna la abolición del derecho probatorio y la vigencia de las reglas generales de la epistemología, mientras que el segundo propone abandonar los esquemas epistemológicos para estudiar la prueba jurídica; pero ambos comparten un claro punto de partida, i.e., el derecho no debe regular la prueba ni las decisiones sobre ésta si no se quiere deslindar completamente la prueba jurídica de la búsqueda de la verdad y de sus patrones epistemológicos.

Pues bien, en lo que sigue pretendo precisamente discutir este preciso punto de encuentro. Sostendré (I) que el derecho necesariamente debe regular (algunos aspectos de) la prueba judicial, aún asumiendo los postulados básicos de la tradición racionalista y (II) que la influencia de Bentham y la tradición racionalista en el derecho probatorio (especialmente anglo-americano) ha tenido éxito especialmente en aquellos puntos más débiles de su pensamiento.

\section{LOS MOMENTOS DE LA ACTIVIDAD PROBATORIA EN EL PROCESO JUDICIAL}

A los efectos aportar nueva luz sobre las exigencias epistemológicas que pueden hacer compatible el derecho probatorio con el objetivo de la búsqueda de la verdad, conviene distinguir claramente tres momentos fundamentales en ese proceso de toma de decisiones sobre los hechos en el proceso judicial. Antes de identificarlos, no obstante, debo advertir que se trata de tres momentos lógicamente distintos y sucesivos, aunque en los procesos de toma de decisiones reales pueden presentarse entrelazados. Se trata de a) la conformación del conjunto de elementos de juicio sobre cuya base se adoptará la decisión; b) la valoración de esos elementos; y c) propiamente, la adopción de la decisión.

de la prueba y otorgar al juez los poderes apropiados para tomar las decisiones necesarias (Bentham, J.: The Rationale of Judicial Evidence, en Id., 1838-43, vol. vi: 151. Vid. También Twining, 1985: 66-75; Postema, 1986: 349; Pardo: 2005: 325) 


\subsection{LA CONFORMACIÓN DEL CONJUNTO DE ELEMENTOS DE JUICIO O PRUEBAS}

En primer lugar, el desarrollo del proceso judicial, a través de la proposición y práctica de las pruebas debe permitir conformar un conjunto de elementos de juicio que apoyen o refuten las distintas hipótesis sobre los hechos del caso. Y aquí se da ya una de las especificidades jurídicas de mayor calado, que puede resumirse en la máxima quid non est in actis non est in mundo. Es decir, a los efectos de la decisión jurídica el conjunto de elementos de juicio que podrá y deberá ser tomado en consideración está formado únicamente por las pruebas aportadas y admitidas al proceso $0^{6}$, no pudiéndose tomar en cuenta, por parte del órgano decisor, aquellas informaciones o elementos de juicio de los que disponga "privadamente» (Stein, 1893); (Damaska, 1986) o aquellos que, habiéndose aportado al proceso han sido excluidos, por ejemplo, por su carácter ilícito. Creo que puede decirse sin excesivo riesgo de error que en todos los demás ámbitos del conocimiento, el conjunto de elementos de juicio que puede y debe ser analizado para adoptar una decisión racional es igual al total de las informaciones disponibles y relevantes para el caso ${ }^{7}$. En el caso de la prueba jurídica, en cambio, el conjunto de elementos a valorar es un subconjunto del conjunto formado por la totalidad de los elementos disponibles: aquéllos de ellos que han sido incorporados al expediente judicial.

De este modo, adquiere una relevancia destacada el estudio de los filtros para la admisión de pruebas en el proceso. Un primer filtro, de orden epistemológico, prescribe la admisión de toda prueba que aporte información relevante sobre los hechos que se juzgan (Bentham, 1823; Taruffo, 1992; Gascon, 1999). Una prueba es relevante si aporta apoyo o refutación de alguna de las hipótesis fácticas del caso a la luz de los principios generales de la lógica y de la ciencia (Twining, 1994). Puede considerarse este filtro, en realidad, como un principio general de inclusión. Funcionaría, así, prescribiendo la admisión de toda prueba relevante que no deba excluirse por aplicación de alguno de los filtros adicionales impuestos por las reglas jurídicas ${ }^{8}$. La diferencia con otros ámbitos de la experiencia es que en esos otros ámbitos es común que ese sea el único filtro. La particularidad jurídica es que se añaden un buen número de reglas jurídicas de exclusión: no se admiten las pruebas obtenidas en violación de derechos fundamentales, no se admiten los testigos de referencia, no se admiten, con salvedades, las pruebas que no puedan practicarse en el curso del proceso con la debida aplicación del principio de contradicción, etc. Además, algunos sujetos cuentan con lo que la doctrina anglosajona denomina «privileges»: esto

\footnotetext{
${ }^{6} \mathrm{~A}$ las que habrá que añadir, en su caso, los hechos admitidos por todas las partes y los denominados «hechos notorios», de los que suele decirse que no necesitan prueba.

${ }^{7}$ Por ello, como he justificado con mayor detalle en Ferrer (2006) no puede explicarse el resultado de la valoración de la prueba en el derecho mediante la noción de convicción judicial o de creencia. La creencia, por decirlo de un modo simple, es siempre all things considered, mientras que el resultado de la valoración de la prueba es siempre contextual: su justificación es relativa a ese conjunto de elementos de juicio.

${ }^{8}$ Este es el punto de vista de Thayer (1898: 266 ss.), del que puede verse una buena exposición en McNamara, 1986. Contra esta tesis de la concepción racionalista de la prueba, puede verse Stein, A., 2005: 108 ss. En mi opinión, como se verá, el análisis de Stein sufre la confusión de no distinguir entre los distintos momentos de la actividad probatoria y, correspondientemente, entre los efectos de la regulación jurídica en cada uno de ellos.
} 
es, se les exime de aportar información relevante para el caso (abogados -respecto de la información obtenida de sus clientes-, sacerdotes -respecto de la información obtenida en confesión-, cualquier sujeto respecto de la información que pueda incriminarle, familiares directos -respecto de informaciones que puedan perjudicar o beneficiar a esos familiares- etc.). Finalmente, pero sin menor importancia, y sin ánimo de exhaustividad, conviene señalar que también los propios plazos procesales juegan un papel de regla de exclusión: se excluye toda información-aunque sea relevante- que se presente mediante pruebas aportadas fuera de los plazos expresamente previstos.

Como ya señaló Bentahm (1823) buena parte de esas reglas que excluyen elementos de juicio lógicamente relevantes se justifican en la protección por parte del derecho de valores distintos a la averiguación de la verdad: la intimidad y otros derechos fundamentales, la autonomía individual, las relaciones familiares, etc. En otros casos, en cambio, se excluyen elementos de juicio por considerarlos de bajo valor epistemológico ante el peligro de que una mala valoración tienda a darles más valor del que tienen. El número y el alcance de las reglas jurídicas que regulan la conformación del conjunto de elementos de juicio varía en función de los distintos ordenamientos y, dentro de ellos, en función de los tipos de proceso y jurisdicción.

\subsection{LA VALORACIÓN DE LOS ELEMENTOS DE JUICIO O PRUEBAS}

A partir de ahí, llegados al momento en que se cierra la composición del conjunto de elementos de juicio que deberán valorarse (con una simplificación quizás excesiva, pero elocuente: una vez declarado el proceso visto para sentencia), se pasa al segundo momento. Debe ahora procederse a la valoración de los elementos de juicio disponibles en el proceso. Si el sistema jurídico establece un régimen de libre valoración de la prueba, entonces deberá valorarse el apoyo que cada elemento de juicio aporta a las hipótesis en conflicto, de forma individual y en conjunto. Con ello, deberá obtenerse un resultado que nos permita saber el grado de confirmación del que dispone cada una de esas hipótesis.

Deben hacerse aquí dos observaciones importantes: en primer lugar, el resultado de la valoración de la prueba es siempre contextual, esto es, referido a un determinado conjunto de elementos de juicio (Mendoca, 1997). Si cambia el conjunto, por adición o sustracción de algún elemento, el resultado puede perfectamente ser otro (Ferrer, 2005). En segundo lugar, la libre valoración de la prueba es libre sólo en el sentido de que no está sujeta a normas jurídicas que predeterminen el resultado de esa valoración. La operación consistente en juzgar el apoyo empírico que un conjunto de elementos de juicio aportan a una hipótesis está sujeta a los criterios generales de la lógica y de la racionalidad, entre otros muchos, (Rescher y Joint, 1959; Damaska, 1986; Twinning, 1994; Taruffo, 1990). Puede advertirse que ese apoyo empírico ofrecerá un grado de corroboración que, como ya señalara Popper, nunca será concluyente, pero, como él 
mismo advierte también "aunque no podamos justificar una teoría (...), podemos, a veces, justificar nuestra preferencia por una teoría sobre otra; por ejemplo, si su grado de corroboración es mayor" (Popper, 1974, p.140).

Si el primero de los momentos, la conformación del conjunto de elementos de juicio, acoge la gran mayoría de especificidades jurídicas, éste segundo momento (siempre que estemos en el ámbito de la libre valoración de la prueba) es el momento de la racionalidad (Thayer, 1898). Se trata aquí, insisto, como en cualquier otro ámbito del conocimiento, de evaluar el apoyo empírico que un conjunto de elementos de juicio aportan a una determinada hipótesis o a su contraria. Si tengo, pues, razón en esto, negar la posibilidad de adoptar decisiones racionales en este momento, a pesar de las especificidades jurídicas que puedan regir en el primero y el tercero de los momentos de la prueba jurídica, supone necesariamente acoger argumentos escépticos generales ante la posibilidad del conocimiento o, más estrictamente, de la decisión racional. En otras palabras: no se puede negar la posibilidad de adoptar decisiones racionales en el ámbito de la prueba jurídica por razones específicamente jurídicas cuando no hay reglas jurídicas que limiten la libre valoración de la prueba.

Así pues, las limitaciones jurídicas que excluyen pruebas relevantes en el momento de la conformación del conjunto de elementos de juicio hacen que el conjunto sea epistemológicamente más rico o más pobre. Las limitaciones temporales, las limitaciones de recursos humanos y materiales, la falta de neutralidad de la intervención de las partes al aportar elementos de juicio, etc., producen, en general, que el conjunto de elementos de juicio disponible sea más pobre. Pero una vez ese conjunto está ya delimitado, la operación de valorar lo que de él se puede racionalmente inferir no difiere en nada de la que se puede realizar en cualquier otro ámbito de la experiencia y está sometida a los controles de la racionalidad general.

\subsection{LA ADOPCIÓN DE LA DECISIÓN SOBRE LOS HECHOS PROBADOS}

Por último, el tercer momento es el que corresponde a la toma de la decisión. La valoración de la prueba habrá permitido otorgar a cada una de las hipótesis en conflicto un determinado grado de confirmación que nunca será igual a la certeza absoluta. Habrá que decidir ahora si la hipótesis $h$ puede o no declararse probada con el grado de confirmación de que disponga. Esto depende del estándar de prueba que se utilice. Así, por ejemplo, es muy usual sostener (especialmente en la cultura jurídica anglosajona) que en el ámbito civil opera el estándar de la prueba prevaleciente, de modo que una hipótesis está probada si su grado de confirmación es superior al de la hipótesis contraria. En cambio, en el ámbito penal, operaría el estándar que exige que la hipótesis esté confirmada más allá de toda duda razonable. Es claro que aquí, de nuevo, la elección de uno u otro estándar es propiamente jurídica y se realiza en atención a los valores en juego en cada tipo de proceso (Stein,2005). Así, puede justificarse la mayor exigencia 
probatoria en los casos penales, por ejemplo, en una especial protección del derecho a la libertad (vehiculada a través del derecho a la presunción de inocencia).

Conviene insistir en que el resultado de la valoración de la prueba que se obtenga en el segundo momento no implica por sí solo nada respecto de la decisión a adoptar. Para ello, es necesaria la intermediación de algún estándar de prueba. Y ni siquiera puede darse por descontado que la hipótesis que haya resultado más confirmada es aquélla que deberá darse por probada. Si se observa bien el funcionamiento del estándar que exige una confirmación de la hipótesis de la culpabilidad, en un proceso penal, más allá de toda duda razonable, éste supone que la hipótesis no se considerará probada aunque disponga de un apoyo empírico mayor que la hipótesis de la inocencia (salvo que ese apoyo ofrezca una corroboración muy alta a la primera), de forma que se presumirá la verdad de la hipótesis menos confirmada (i.e., la de la inocencia).

\section{LA CRÍTICA DE STEIN A LA TRADICIÓN RACIONALISTA}

En el apartado anterior he señalado la vigencia, con algunas excepciones, del principio general de que cualquier elemento de juicio relevante para la adopción de una decisión debe ser admitido como prueba en el proceso judicial. Este principio general se justifica epistemológicamente en la medida en que garantiza la mayor probabilidad de que los enunciados que se declaren probados coincidan con la verdad.

Ahora bien, la averiguación de la verdad es un fin en algún sentido prioritario del proceso en materia de prueba ${ }^{9}$, pero no es en absoluto el único. La celeridad en la toma de decisiones, la protección de derechos fundamentales, la protección de secretos de estado, el secreto de las relaciones abogado-cliente, etc., son también fines habitualmente reconocidos en la gran mayoría de ordenamientos jurídicos (Liebman, 1955, p. 276-277; Rescher y Joynt 1959 p.568; Weinstein, J.; 1966, p. 241 .; Damaska, 1986, p.160; 1997 p.12; Posner, 1990, p.206; y , Taruffo, 1992; p.336-337; Stein, A., 2000, p.110; entre otros muchos). Por ello para garantizar la obtención de esas otras finalidades, los ordenamientos establecen reglas jurídicas procesales (aunque no sólo procesales) que funcionan como un filtro de admisibilidad de la prueba, adicional y posterior al juicio de relevancia. Resulta claro que la racionalidad instrumental de esas reglas jurídicas no puede ser evaluada teniendo en mente la finalidad de la averiguación de la verdad, sino la finalidad a la que en cada caso respondan. Pero ello significa también que las reglas que persiguen la garantía de esos otros fines del proceso o del derecho pueden imponer algún sacrificio epistemológico, en el sentido de que pueden ser contraproducentes para el objetivo de la averiguación de la verdad. En general es así, en efecto, cada vez que la protección o maximización de esos otros fines del proceso supone la exclusión de pruebas relevantes para el caso que debe decidirse. Esto no implica que sea imposible alcanzar una determinación verdadera

\footnotetext{
${ }^{9}$ Se trata seguramente del único fin funcionalmente necesario para que sea posible la aplicación del derecho y, por ello, para que el derecho como instrumento de control social pueda también funcionar. 
de los hechos ocurridos, sino, más modestamente, que las probabilidades de que ello ocurra, dado que el conjunto de elementos de juicio será más pobre, serán también más bajas. Y siendo consciente de ese coste, el legislador deberá decidir en cada caso de conflicto entre la maximización de los fines epistemológicos y de cualquier otro que se quiera proteger, en qué medida se sacrifican unos y otros. (Wroblewky, 1981)

Para evaluar en cada caso si este tipo de reglas de exclusión están justificadas, habrá que juzgar su racionalidad teleológica atendiendo a su adecuación como medios para alcanzar los fines a los que responden. Y, además, evaluar, dado que entran en conflicto con la finalidad de la averiguación de la verdad, si están disponibles otros medios para alcanzar esos fines que no conlleven este conflicto (evitando así la regla de exclusión).

Ahora bien, resulta evidente que cualquier regla de exclusión de este tipo supone el rechazo de una prueba que ha pasado el filtro de la relevancia y que, por tanto, tiene a su favor el principio general de inclusión de toda prueba relevante. Es decir, esas reglas de exclusión suponen que existe un conflicto entre distintos fines considerados valiosos por el derecho. Así, por ejemplo, la averiguación de la verdad versus la inviolabilidad de la correspondencia. La justificación de la regla de exclusión no es, en esos casos, sólo una cuestión de racionalidad teleológica. Tiene también un aspecto valorativo de gran importancia. Se trata de la ordenación de los valores que permita decidir por uno o por otro en caso de conflicto. Y esta ya no es una cuestión de racionalidad sino valorativa. El legislador deberá adoptar una decisión respecto de la ordenación de los valores en conflicto y esa decisión sólo podrá ser juzgada desde patrones valorativos.

AlexStein (2005) ha insistido mucho en que no se da aquí un conflicto entre la epistemología (entendida como actividad neutral desde el punto de vista valorativo) y ciertos valores sociales o morales que el derecho pretende proteger, sino, en último término, como he señalado, un conflicto entre distintos valores, que el legislador deberá ordenar si quiere resolver el conflicto. Esto es, la averiguación de la verdad es también un valor para el derecho. A partir de esta constatación, Stein elabora un doble argumento contra el que denomina «movimiento abolicionista» del derecho probatorio, que, liderado entre otros por Bentham y Thayer, sostiene que la valoración de la prueba es una cuestión puramente epistemológica respecto de la que el derecho haría bien en no inmiscuirse:

1) En primer lugar, la epistemología sólo nos sirve para determinar el grado de probabilidad de que una hipótesis sobre los hechos sea verdadera, pero nada nos dice sobre el punto en que esa probabilidad es suficiente para aceptar como verdadera la hipótesis. Es decir, la epistemología no puede determinar los estándares de prueba (Stein, 2005). Establecer el umbral a partir del cual el nivel de corroboración de una hipótesis es suficiente supone tomar una decisión sobre el reparto del riesgo de error. Así, en efecto, si el estándar de prueba en el proceso penal es más exigente se producirán menos condenas falsas y más absoluciones falsas, mientras que el efecto será exactamente el contrario si el estándar 
se sitúa en un punto menor de exigencia. Pues bien, esa es una decisión que queda absolutamente en el ámbito político-moral sobre el que la epistemología no tiene nada que decir. Para ello es necesario, pues, que se pronuncie el derecho.

2) En segundo lugar, como hemos visto, el denominado principio de inclusión impone la admisión de toda prueba relevante para la decisión sobre los hechos que debe tomarse en el proceso. $\mathrm{O}$, dicho de otro modo, el principio implicaría, tomado estrictamente, la derogación de las reglas probatorias que excluyen pruebas relevantes. Esta es la clásica formulación de Bentham (1827) y tiene su fundamento en que la probabilidad de alcanzar una decisión correcta respecto de la verdad sobre los hechos aumenta en la medida en que lo hace la información sobre lo ocurrido. La riqueza o peso del conjunto de elementos de juicio sobre el que se adopte la decisión estaría en directa relación con la probabilidad de que la decisión sea correcta. La objeción de Stein (2005) en cambio, ataca precisamente el fundamento del principio de inclusión:

Mientras la información de que disponga el decisor sobre los hechos siga siendo incompleta, la adquisición de información adicional con credenciales inciertas puede no mejorar su posición epistémica. La llegada de nueva información podría sólo sustituir el riesgo de error existente hasta el momento por un nuevo riesgo de error, vinculado a la credibilidad de la nueva información. Más aún, no hay garantía de que el nuevo riesgo de error sea menor que el anterior.

(...)

« (...) [M]i tesis simplemente refuta la intuitiva (pero falaz) idea de que el aumento de información necesariamente produce mayor exactitud en la determinación de los hechos (p.123).

Pues bien, creo que el desafío de Stein al movimiento abolicionista ${ }^{10}$ debe ser cuidadosamente analizado. Tiene razón el autor cuando plantea que la averiguación de la verdad es un valor más que el derecho persigue y que puede entrar en conflicto con otros valores asumidos por el mismo derecho ${ }^{11}$. En esos casos, evidentemente, estaremos ante un conflicto de valores que habrá que resolver. Y la decisión que se adopte no será en ningún caso informada por la epistemología sino por la política y la moral. Sin embargo, conviene tener en cuenta que, en mi opinión, el valor o la finalidad de la averiguación de la verdad no tiene estructuralmente la misma posición que los demás. Como ya he defendido en otro lugar Ferrer (2007), el objetivo institucional de la prueba en el proceso es la averiguación de la verdad. Y ello no puede ser de otra manera, puesto que ese objetivo es estructuralmente necesario para que funcione el propio derecho como mecanismo de motivación de la conducta ${ }^{12}$. Sólo si las consecuencias jurídicas

\footnotetext{
${ }^{10}$ Como se habrá advertido, ese movimiento teórico coincide fundamentalmente con las tesis de la concepción racionalista de la prueba, mencionada en el primer epígrafe, y que otros autores han denominado movimiento «Free proof».

${ }^{11}$ Punto este que no niega en absoluto la tradición racionalista (que coincide básicamente con lo que Stein denomina «movimiento abolicionista»). Vid. Anderson-Schum-Twining, 1991: 79-80.

${ }^{12}$ No pretendo entrar aquí en el debate de las funciones del derecho, que es muy complejo e innecesario para el argumento que
} 
previstas por el derecho para acciones determinadas se aplican efectivamente a esas acciones (idealmente siempre a esas acciones y nunca a otras), los ciudadanos tendrán motivos (jurídicos) para actuar conforme a lo prescrito por el derecho y este podrá cumplir su función de mecanismo de resolución de conflictos. Esta faceta estructural no es compartida por otros valores con los que la averiguación de la verdad puede entrar en conflicto. Un sistema jurídico puede funcionar perfectamente sin asumir el valor de la inviolabilidad del domicilio o de las comunicaciones, por ejemplo. Sería este un sistema jurídico indeseable por otras razones, pero no hay nada que impida que un sistema así sea perfectamente efectivo y eficiente en el cumplimiento de su función motivadora de la conducta. En cambio, el sistema no puede prescindir de la averiguación de la verdad como objetivo institucional del proceso ( $y$, por tanto, de la aplicación del derecho), puesto que de otro modo el sistema colapsaría. Esto no le otorga un mayor valor moral a la averiguación de la verdad, pero muestra que en el conflicto entre ese objetivo del derecho y otros posibles objetivos, el primero tiene una preferencia estructural que hace que no pueda ceder siempre.

Dicho esto, creo que el primer argumento de Stein es perfectamente correcto. Efectivamente, la decisión sobre el nivel de suficiencia probatoria no es en absoluto epistemológica. La epistemología nos puede ayudar a delinear un estándar de prueba que refleje correctamente el nivel de suficiencia probatoria que se haya decidido adoptar, pero no nos dice nada sobre el nivel mismo. Esa es una decisión política. Esto, de todos modos, no es específico del derecho. También la farmacología, la historia, la epidemiología o la astronomía necesitan de estándares de prueba claramente definidos. Y tampoco la epistemología sirve en esos campos para definir el nivel de exigencia del estándar. Se trata también en esos casos de decisiones sociales (i.e., de la comunidad científica) que toman en cuenta también los bienes en juego. No es extraño, pues, que el estándar de prueba farmacológico sea mucho más elevado que el de la historia. Y ello no por razones epistemológicas, sino por el coste del riesgo de error sobre los bienes en juego ${ }^{13}$. La única diferencia en el caso del derecho es que tenemos mecanismos institucionales para adoptar las decisiones sobre los estándares de prueba.

En cambio, el segundo argumento de Stein, que afecta más centralmente al objeto de discusión de este epígrafe, me parece erróneo. Desde luego, el aumento de información

estoy sosteniendo. Basta, por el momento, con aceptar que cualquiera que sea la función que se pretenda atribuir al derecho, el cumplimiento de esa función exige que el derecho tenga un alto grado de eficacia como mecanismo de guía de la conducta (HART, 1994: 248-9). Y para que esa eficacia sea posible, pretendo mostrar que es necesario que en la aplicación del derecho se imponga la consecuencia jurídica prevista por las normas generales a los casos en que se haya dado el supuesto de hecho previsto también por las normas (y no se imponga a los casos en que el supuesto de hecho no se dé). Si esto es así, se obtiene ya que el proceso judicial, por lo que atañe a la prueba, no puede tener otro objetivo fundamental que la averiguación de la verdad.

${ }^{13}$ No somos indiferentes ante los falsos positivos o falsos negativos en investigación farmacológica, de modo que preferimos un falso negativo (un medicamento que no supere el estándar y que, en cambio, sea beneficioso para la salud) a un falso positivo (un medicamento que supere el estándar y que, en cambio, sea perjudicial para la salud). Por ello, se define un estándar de prueba muy exigente que distribuye desigualmente los riesgos de error. En cambio, sí somos indiferentes ante la distribución de errores entre los falsos positivos y negativos en investigación histórica, por lo que el estándar de prueba se sitúa en la preponderancia de la prueba (es decir, en la exigencia de que la probabilidad de una hipótesis sea mayor que la de su negación). 
relevante para la adopción de una decisión no necesariamente produce un aumento en la exactitud de la decisión. En ello tiene razón Stein. Pero no es esto lo que necesita el movimiento abolicionista o la tradición racionalista para sostener que tendencialmente toda prueba relevante debe ser admitida ( $y$, por tanto, deben abolirse las reglas de exclusión correspondientes). El fundamento necesario para esa tesis es algo más débil que el rechazado por Stein: que el aumento de información relevante aumenta, ceteris paribus, la probabilidad de que se adopte una decisión adecuada materialmente, esto es, que se declaren probados enunciados verdaderos sobre los hechos. Afirmar únicamente que una decisión es adecuada o correcta es ambiguo, por cuanto podría significar que es la decisión fundamentada en el conjunto de elementos de juicio disponible (con independencia de su valor de verdad) o que es una decisión cuyo contenido se corresponde con la verdad de lo ocurrido (Ferrer, 2005). Se puede distinguir así entre la validez y la verdad de la decisión (Laudan, 2006). Una decisión sobre los hechos será válida si está fundada en los elementos de juicio disponibles. Será, en cambio, verdadera si se corresponde con los hechos del mundo. Pues bien, el aumento de la riqueza del conjunto de elementos de juicio (o de la información, en palabras de Stein) aumenta la probabilidad de que la decisión válida sea también verdadera. Y ello es fundamento suficiente para justificar el principio de inclusión ${ }^{14}$.

\section{RECONSIDERANDO LAS TESIS DE PARTIDA}

Una vez analizados los distintos momentos de la actividad probatoria en el proceso judicial, estamos en condiciones de revisitar las tesis de Bentham presentadas al inicio del trabajo y evaluar la incidencia, real y/o deseable, que tienen en nuestros sistemas procesales.

En este sentido, Twining (1994) sostiene que las tesis abolicionistas del derecho probatorio propugnadas por Bentham han tenido éxito (en el mundo anglosajón) en lo que hace a la inexistencia de reglas sobre la valoración de la prueba y, en cambio, no lo han tenido tanto en lo que hace a las reglas de exclusión de pruebas relevantes. Desde un punto de vista estrictamente epistemológico, creo que puede decirse que, de ser así, el resultado ha sido el inverso al deseable.

En efecto, en los sistemas jurídicos anglosajones el derecho probatorio es, fundamentalmente, un conjunto a veces muy prolífico y casuístico de reglas sobre la admisibilidad y la exclusión de la prueba. En cambio, prácticamente nada se dice sobre la valoración de la prueba y las reglas que regulan el momento de la adopción de la decisión sobre los hechos probados son extraordinariamente vagas, al punto de resultar poco menos que inservibles.

\footnotetext{
${ }^{14}$ La crítica de Stein tiene un argumento más que debe ser analizado. Sostiene el autor que el aumento de información puede ser contraproducente para la corrección de la decisión si hay dudas sobre las credenciales de la información, esto es, sobre su fiabilidad. Desde luego, ello puede suceder. En todo caso, en línea de principio, la respuesta es que en ese supuesto lo que corresponde es un nuevo aumento de la información que nos permita evaluar la fiabilidad de la nueva prueba adquirida. Desde luego, en algún lugar hay que poner límites a éste continuo para evitar el regreso al infinito.
} 
En el momento de la valoración de la prueba, el juez, jurado o tribunal deberá evaluar qué hipótesis de las diversas en conflicto en el proceso, tiene un más alto grado de contrastación. Ésta es una labor comparativa entre las distintas hipótesis, a la luz de los test realizados y presentados como pruebas por las partes, peritos, etc., u ordenadas por el propio juez si el sistema se lo permite. Pero el resultado de esta valoración no podrá ser más que una ordenación del nivel de contrastación de las distintas hipótesis. Para llegar a la conclusión que atribuye a una de ellas la categoría de hecho probado es necesario contar con un criterio que nos indique a partir de qué nivel de contrastación vamos a considerar probada la hipótesis. Necesitamos un estándar de prueba ${ }^{15}$.

Como he señalado anteriormente, esto no es distinto de lo que ocurre en cualquier otro ámbito de la experiencia. En paleontología, medicina, historia, farmacología, química orgánica o física nuclear son necesarios también estándares de prueba que permitan decidir cuándo una hipótesis en esos ámbitos de investigación puede ser considerada probada. En todos ellos, son las respectivas comunidades científicas las que, de manera normalmente informal y no institucionalizada, adoptan un estándar de prueba atendiendo a la ratio entre errores positivos y negativos que se consideran aceptables en esos ámbitos. Y para ello la epistemología no impone más que metodologías de formulación del estándar, no el estándar mismo. No es raro, pues, que eso sea necesario también en el ámbito jurídico como bien señala Stein (2005). La especificidad, como he indicado ya con antelación, es el carácter institucionalizado de la toma de decisiones jurídicas. Y, por razones democráticas, tampoco es raro que esa decisión corresponda adoptarla a los legisladores (para cada tipo de proceso jurídico).

Si esto es así, pues, conviene matizar las exigencias de las tesis abolicionistas: el derecho no puede dejar de regular todos los aspectos de la prueba en el proceso para que rijan sólo criterios epistemológicos por la sencilla razón de que la epistemología no es suficiente para adoptar decisiones (ni pretende serlo). En cambio, sí sería la epistemología una guía suficiente para el momento de la conformación del conjunto de elementos de juicio (si el valor de la búsqueda de la verdad fuera el único que nos guiara o prevaleciera siempre en caso de conflicto con otros, cosa que no ocurre en ningún ordenamiento). Y también, especialmente, en el momento de la valoración de la prueba, si estamos bajo el régimen de la libre valoración de la prueba.

La situación en el ámbito de los países de civil law es bastante distinta. Damaška (1997) sostiene la tesis, muy atendible, de que la falta de una exigencia jurídica de motivación del juicio sobre los hechos en los ordenamientos de common law explica la especial proliferación en ese ámbito de reglas jurídicas que excluyen pruebas que se considera que aportan información poco fiable. El objetivo es evitar que en la valoración de las

${ }^{15}$ Esto es así incluso en el caso en que se adopten las tesis de la inferencia a la mejor explicación, puesto que en este caso, sencillamente, el estándar de prueba indicaría que se acepte como probada la hipótesis relativamente mejor contrastada (aunque su nivel de contrastación sea bajo), por el hecho de que es la mejor de que disponemos. 
pruebas se les otorgue un peso excesivo ${ }^{16}$. En algún sentido, estas reglas pretenden garantizar ex ante una mayor racionalidad general de las decisiones sobre los hechos a costa de excluir elementos de juicio que, aun con un valor relativamente escaso, pudieran aportar informaciones relevantes. En los ordenamientos de civil law, en cambio, el control de la racionalidad de la decisión se realizaría ex post mediante el control de la motivación Igartua, 2003). Así, por ejemplo, ante una prueba o un tipo de prueba de muy baja fiabilidad, el control de racionalidad podrá funcionar a posteriori siempre que se exija al decisor sobre los hechos probados que justifique porqué ha declarado probados esos hechos y cuál ha sido el apoyo empírico en el que ha basado su decisión. Podrá comprobarse, entonces, si el decisor ha otorgado un peso excesivo a una prueba cuya fiabilidad resulte cuestionada ${ }^{17}$. En cambio, si no se exige motivación de la decisión sobre los hechos, el único modo de asegurar normativamente que no se tome la decisión sobre la base de pruebas poco o nada fiables es, directamente, excluirlas del conjunto de elementos de juicio disponibles ${ }^{18}$.

Pero el problema en los sistemas de civil law respecto de los estándares de prueba es el mismo, su práctica inexistencia. Tomemos como ejemplo el proceso penal: la tradicional apelación a la íntima convicción o la progresiva incorporación (por vía legislativa o jurisprudencial) del "más allá de toda duda razonable" propio de los sistemas anglosajones (McCormick, 1954, p.445-449) a los sistemas continentales ${ }^{19}$ no satisface las necesidades en absoluto, puesto que deja la decisión en manos de la subjetividad del decisor y no permite ningún control intersubjetivo. Como ha mostrado muy convincentemente Laudan (2003-2006, p.29-62) si no disponemos de un criterio que nos indique las condiciones en que la duda será razonable (que no podrá ser cuantitativo), no podremos decir en ningún caso que disponemos de un estándar de decisión objetivo (o al menos intersubjetivo) y cognoscible a priori. Y esto producirá serios problemas para la finalidad motivadora de la conducta propia del derecho: si no disponemos de un estándar de prueba cognoscible a priori, las decisiones judiciales no podrán ser previsibles, lo que impide la adaptación estratégica de la conducta a la decisión prevista ${ }^{20}$.

\footnotetext{
${ }^{16}$ Pero no es éste el único motivo que explica la tradicional proliferación de reglas de exclusión de pruebas en los sistemas de common law. Hay que añadir también, por ejemplo, la presencia generalizada de la institución del jurado compuesto exclusivamente por legos (Damaška, 1997: 53-4) como órgano decisor sobre los hechos del caso y al que se pretende instruir detalladamente sobre las reglas que deben guiar su función.

17 Esto, por supuesto, está también vinculado a la existencia de un sistema de recursos para la revisión de la decisión adoptada (que sea, además, simétrico, i.e., igual para todas las las partes).

18 A pesar de ello, en ausencia de motivación detallada no hay forma de comprobar las razones que se han usado para adoptar la decisión. Podrá solo controlarse si, a la luz de los elementos de juicio disponibles en el proceso, esa decisión resulta suficientemente justificada o no, realizando para ello una nueva valoración de los mismos.

19 El uso de esta fórmula se ha expandido en los últimos años también en muchos países de tradición romano-germánica. Es el caso, por ejemplo, de Italia y España. Vid., al respecto, STELLA (2001: 195-218) para el caso italiano y FERNÁndez LóPEZ (2005: 199-208) para el caso español. También el Tribunal Europeo de Derechos Humanos ha manifestado que el derecho a la presunción de inocencia establecido en el artículo 6.2 del CEDH (Convenio Europeo para la Protección de los Derechos Humanos y Libertades Fundamentales) implica que sobre la acusación pesa la carga de la prueba más allá de toda duda razonable (STRDH de 6 de diciembre de 1988: caso Barberá, Messegué y Jabardo v. España). En mi opinión, no obstante, la presunción de inocencia como regla de juicio establece únicamente que en caso de duda se presuma la inocencia del acusado, pero no implica ningún estándar de prueba específico. 0 , dicho de otro modo, el derecho a la presunción de inocencia es compatible con estándares de prueba distintos, que expresan diversos niveles de exigencia probatoria.

20 Por esta razón, no puedo estar de acuerdo con autores como Stoffelmayr-Seidman Diamond (2000: 781) y Lillquist (2002: 146 ss.,
} 
En otro lugar he propuesto una formulación del estándar de prueba penal (para la decisión final sobre los hechos probados de la hipótesis acusatoria) que pretende salvar los inconvenientes señalados. No abordaré aquí su justificación ni siquiera su explicación detallada, pero sí lo formularé a los efectos de facilitar la comprensión sobre las exigencias mencionadas.

Así, según este estándar de prueba, para considerar probada la hipótesis de la culpabilidad deberían darse conjuntamente las siguientes condiciones:

1) La hipótesis debe ser capaz de explicar los datos disponibles, integrándolos de forma coherente, y las predicciones de nuevos datos que la hipótesis permita formular deben haber resultado confirmadas.

2) Deben haberse refutado ${ }^{21}$ todas las demás hipótesis plausibles explicativas de los mismos datos que sean compatibles con la inocencia del acusado, excluidas las meras hipótesis ad hoc 22 .

La falta de estándares de prueba claros y objetivos no puede atribuirse, claro está, a los supuestos efectos perniciosos de las tesis de Bentham y de la tradición racionalista o abolicionista. Pero esa tradición, y el importantísimo influjo de Bentham en particular,

162 ss.), que consideran justificable o incluso adecuado disponer de un estándar de prueba "flexible" para que pueda ser adaptado por el juzgador a las circunstancias del caso concreto e incluso del acusado. Por ello, sostienen estos autores, puede ser conveniente una formulación extremadamente vaga y no definida del estándar de prueba (como la de "más allá de toda duda razonable"), que permita esa flexibilidad. En mi opinión, ello conlleva necesariamente la imposibilidad del control sobre la aplicación del estándar de prueba y, por consecuencia, del cumplimiento del derecho a la presunción de inocencia.

21 Conviene aclarar el sentido en que uso el término "refutación" en este trabajo para evitar una problemática confusión: entiendo la refutación como el rechazo de una hipótesis derivado del incumplimiento de las predicciones que pueden formularse a partir de ellas. Hay que advertir, no obstante, que el incumplimiento de una predicción formulable a partir de una hipótesis no conlleva necesariamente la refutación de la hipótesis. Al respecto puede verse FERRER (2007: 132) y LeITER (1997: 807-808). Por ello, contra lo que pudiera parecer (y sostuvo Popper), no hay asimetría entre la corroboración de una hipótesis y su refutación: ambos son procesos graduales y probabilísticas.

22 Este es el único requisito planteado por AlLEN (1994: 604), aunque él no hace la advertencia de la exclusión de las hipótesis ad hoc. Siendo así, se presenta el problema de que siempre es posible construir una historia coherente que dé cuenta a posteriori de los datos disponibles. El acusado podrá sostener, por ejemplo, la hipótesis del complot en su contra, que quizás resulte irrefutable. Pero es una hipótesis posible. A ALLEN le queda como salida hacer más fuerte el requisito de la plausibilidad, de modo que no excluya únicamente las hipótesis incoherentes o incompatibles con nuestro conocimiento del mundo, pero entonces éste se convierte en un requisito tan extremadamente vago que quizás sea inservible. Para evitar este problema, ALLEN y PARDo (2007: 135-136) han propuesto recurrir a la teoría de la inferencia a la mejor explicación, como medida de la plausibilidad. En términos muy generales puede decirse que, según este modelo, una hipótesis será plausible -y, por ello, aceptable como probada en un procedimiento- si es la que mejor explica los hechos conocidos del caso. El modelo de la inferencia a la mejor explicación, basado en el razonamiento abductivo, se remonta a la obra de HARMAN (1965) y ha tenido en los últimos años algunos defensores de su aplicación al razonamiento probatorio jurídico (por todos, AMAYA, 2009). Desde luego, el modelo requeriría de un análisis detallado que no puedo realizar aquí. Sin embargo, conviene señalar dos problemas que a mi entender lo hacen difícilmente utilizable como criterio de decisión para el proceso penal (i.e., como modo de formular su estándar de prueba): 1) en muchas ocasiones, la mejor explicación disponible de los hechos del caso es, sin embargo, una mala explicación. Que sea la mejor explicación depende sólo de una comparación con las otras explicaciones o hipótesis consideradas. Y, de ese modo, por ejemplo, puede suceder que la hipótesis de la acusación sea una mejor explicación de los hechos que la hipótesis de la defensa, pero que a pesar de ello no sea una buena explicación de los mismos. De este modo, usar el modelo de la inferencia a la mejor explicación como forma de fijar el estándar de prueba penal haría de éste un estándar muy débil. 2) El segundo problema se presenta cuando las hipótesis explicativas en conflicto, tanto de la acusación como de la defensa, son buenas. En ese caso, aunque la hipótesis de la acusación sea mejor, no parece adecuado concluir con la aceptación de la hipótesis acusatoria, salvo que de nuevo se debilite mucho el estándar. Sobre estos y otros problemas de la teoría de la inferencia a la mejor explicación aplicada al razonamiento probatorio, vid. LAUDAN, 2007. 
han contribuido en gran medida a que se no se advirtiera tan importante carencia durante los dos últimos siglos ${ }^{23}$. Por el contrario, en los últimos años se está produciendo una reivindicación de las tesis benthamianas, especialmente en países de civil law, por lo que atañe al momento de la conformación del conjunto de elementos de juicio sobre cuya base se deberá tomar la decisión, así como la progresiva derogación de muchas reglas de prueba tasada, que inciden en el segundo de los momentos de la actividad probatoria (el de su valoración). Y esta sí parece una influencia fructífera.

\section{REFERENCIAS BIBLIOGRÁFICAS}

Allen, R.J. (1994). Factual Ambiguity and a Theory of Evidence, Northwestern University Law Review, No.88

Allen, R.J. y Pardo, M.S. (2007). The Problematic Value of Mathematical Models of Evidence", Journal of Legal Studies, No.36

Amaya, A., (2009). In reference to the Best Legal Explanation. Legal Evidence and Proof: Statistics, Stories, Logic, H. Kaptein, H. Prakken, and B. Verheij eds., Aldershot: Ashgate, Prensa.

Anderson, T., Schum, D. y Twining, W. (1991). Analysis of Evidence. USA, New York: Cambridge University Press, 2005.

Bayón, J.C. (2009). Epistemología, moral y prueba de los hechos: hacia un enfoque no benthamiano. Analisi e diritto (en prensa). Citado por el manuscrito presentado en el XIV congreso italo-español de teoría del derecho, Girona, 2008.

Bentham, J. (1823). Traité des preuves judiciares, translated. Dumont, Paris: Bossages fréres. Citado por la traducción de M. Ossorio: Tratado de las pruebas judiciales, Comares, Granada, 2001.

(1827). Rationale of Judicial Evidence, 7 vols., editado por Stuart Mill, J. Citado por la edición incluida en Bentham, 1838-43, vols. VI y VII.

\footnotetext{
23 Bayón (2009: 18) argumenta convincentemente que la preocupación de Bentham estaba centrada en la minimización de errores y no en su distribución. Si esto es así, según Bayón, bastarían reglas epistemológicas para tomar la decisión (que debería coincidir con la hipótesis mejor fundada -lo que para Bayón nos lleva a la inferencia a la mejor explicación- y no serían necesarias ulteriores reglas socio-políticas que establezcan estándares de prueba. Otra cosa sucedería si estuviéramos interesados no sólo (o no principalmente) en la minimización de errores sino en su distribución. Por supuesto, para distribuir los riesgos de error entre las partes en conflicto es necesario introducir una regla que no podrá ser nunca epistemológica. La cuestión es que, en el derecho, no distribuir los riesgos de error desigualmente entre las partes es también tomar una decisión sobre la distribución de riesgos de error. Así es, por ejemplo, se sostiene habitualmente, en el proceso civil, donde imperaría la regla de la preponderancia de la prueba (que distribuye igualitariamente los riesgos de error entre las partes). Y por ello se considera también en el ámbito civil que esa regla constituye un estándar de prueba. La inferencia a la mejor explicación no opera tampoco, en mi opinión, en el ámbito de la jurisdicción civil puesto que, también allí, la mejor explicación puede no superar el estándar de la preponderancia de la prueba (si no se acepta la aplicación del principio del cálculo de probabilidades de la complementariedad). En todo caso, no me resulta posible aquí desarrollar y justificar con detalle estas tesis.
} 
(1838-43). The Works of Jeremy Bentham, 11 vols., ed. por J. Bowring, Bristol: Thoemmes Press, 1995.

Carnelutti, F. (1947). La prova civile. Italia, Roma: Edizioni dell'Ateneo.

Damaška, M. (1986). The Faces of Justice and State Authority: A Comparative Approach to the Legal Process, New Haven-London: Yale University Press.

(1997). Evidence Law Adrift, New Haven-London: Yale University Press.

Fernández López, M. (2005).Prueba y presunción de inocencia. España, Madrid: lustel.

Ferrer Beltrán, J. (2005). Prueba y verdad en el derecho, 2a ed., Madrid: Marcial Pons. (2006). Legal Proof and Fact Finders' Beliefs. Legal Theory, 12.

(2007). La valoración racional de la prueba, Madrid-Barcelona-Buenos Aires: Marcial Pons.

Gascón, M. (1999). Los hechos en el derecho. Bases argumentales de la prueba. España, Madrid: Marcial Pons.

Harman, G. (1965). The inference to the best explanation. Philosophical Review, 74.

Hart, H.L.A. (1982). Essays on Bentham, Oxford: Oxford University Press.

(1994). "Postscript", en Hart, H.L.A. The Concept of Law, 2a ed., Oxford: Oxford University Press.

Igartua, J. (2003). La motivación de las sentencias, imperativo constitucional, España, Madrid: Centro de Estudios Políticos y Constitucionales.

Keeton, G.W. y Marshall, O.K. (1948). Bentham's Influence on the Law of Evidence.

Keeton, G.W. y Schwarzenberger, G. (eds.). Jeremy Bentham and the Law . Ingland, London: Stevens.

Laudan, L. (2003). Is Reasonable Doubt Reasonable?. Legal Theory, 9.

(2006). Truth, Error, and Criminal Law. An Essay in Legal Epistemology, USA, Cambridge: Cambridge University Press. 
(2007). "Strange bedfellows: inference to the best explanation and the criminal standard of proof" en International Journal of Evidence and Proof, 11.

Leiter, B. (1997). The Epistemology of Admissibility: Why Even Good Philosophy of Science Would Not Make for Good Philosophy of Evidence. Brigham Young University Law Review, no. 4,

Liebman, E.T. (1955). Manuale di diritto processuale civile, Milano: Giuffrè. Citado por la traducción castellana de Sentís Melendo, S.: Manual de derecho procesal civil, Buenos Aires: EJEA, 1980.

Lillquist, E. (2002). Recasting Reasonable Doubt: Decision Theory and the Virtues of Variability. U.C. Davis Law Review, 36, no 1.

McCormick, Ch.T. (1954). On Evidence, 4a ed., J.W. Strong. St. Paul (Minn.): West Publishing Co., 1992.

McNamara, Ph. (1986). The Canons of Evidence. Rules of Exclusion or Rules of Use?. Adelaide Law Review, 10.

Mendonca, D. (1997). Interpretación y aplicación del derecho. España, Almería: Servicio de publicaciones de la Universidad de Almería.

Moreso, J.J. (1992). La teoría del derecho de Bentham. España, Barcelona: PPU.

Pardo, M. (2005). The field of Evidence and the Field of Knowledge. Law and Philosophy, no 24 , vol. 5 .

Popper, K. (1974). Unended Quest. An Intellectual Autobiography. The Library of Living Philosophers. Citado por la traducción castellana de GARCÍA TREVIJANO, C. (2002). Búsqueda sin término. Una autobiografía intelectual, España, Madrid: Tecnos,

Posner, R.A. (1990). The Problems of Jurisprudence. USA, Cambridge (Mass.): Harvard University Press.

Postema, G.J. (1986). Bentham and the Common Law Tradition, USA, Oxford: Oxford University Press.

Rescher, N. y Joynt, C.B. (1959). Evidence in History and in the Law. The Journal of Philosophy, vol. LVI, 13.

Sentís Melendo, S. (1979). La prueba. Argentina, Buenos Aires: EJEA. 
Stein, A. (2005). Foundations of Evidence Law. USA, Oxford: Oxford University Press.

Stein, F. (1893). Das private Wissen des Richters. Untersuchungen zum Beweisrecht beider Prozesse. Citado por la traducción castellana Oliva, A. (1990). El conocimiento privado del juez. España, Madrid: Centro de Estudios Ramón Areces.

Stella, F. (2003). Giustizia e modernità. La protezione dell'innocente e la tutela delle vittime. Italia, Milano: Giuffrè.

Stoffelmayr, E. y Siedman Diamond, S. (2000). The Conflict between Precision and Flexibility in Explaining 'Beyond a Reasonable Doubt'. Psychology, Public Policy and Law, 6.

Taruffo, M. (1990). Modelli di prova e di procedimento probatorio. Rivista di diritto processuale, $\mathrm{XLV}, \mathrm{n}=2$.

(1992). La prova dei fatti giuridici. Milano: Giuffrè.

Thayer, J.B. (1898). A Preliminary Treatise on Evidence at the Common Law. USA, Boston: Elibron Classics.

Twining, W. (1985). Theories of Evidence: Bentham \& Wigmore, Stanford, USA, California: Stanford University Press.

Rethinking Evidence. Exploratory Essays. (1992). Evanston, Illinois: Northwestern University Press.

Weinstein, J.B. (1966). Some difficulties in devising rules for determining truth in judicial trials, Columbia Law Review, vol. 66, p.2. Reeditado en Twining, W. y Stein, A. (eds.): Evidence and Proof, Aldershot: Dartmouth, 1992.

Wróblewski, J. (1981). La prueba jurídica: axiología, lógica y argumentación, en Wróblewski, J.: Sentido y hecho en el derecho, San Sebastián, España: Servicio editorial de la Universidad del País Vasco, 1989. 